\title{
Lowest Weight: Reactive Clustering Algorithm for Adhoc Networks
}

\author{
Mohamed Elhoucine Elhdhili, Lamia Ben Azzouz, and Farouk Kamoun
}

\author{
CRISTAL, Ecole Nationale des Sciences de l'Informatique, University of Manouba Tunisia \\ Mohamed.Elhdhili@cristal.rnu.tn, \\ \{Lamia.Benazzouz, Farouk.Kamoun\}@ensi.rnu.tn
}

\begin{abstract}
In this paper, we address clustering in ad hoc networks. Ad hoc networks are a wireless networking paradigm in which mobile hosts rely on each other to keep the network connected without the help of any pre-existing infrastructure or central administrator. Thus, additional features pertinent to this type of networks appeared. In fact, centralized solutions are generally inadaptable due to the need for cooperative network operations. To ensure efficient, tolerant and durable cooperative operations, nodes need to organize themselves. Clustering is an organization method which consists in grouping the nodes into clusters (groups) managed by nodes called clusterheads. In this paper, we present existing clustering algorithms and propose a new solution inspired from two of these algorithms (Lowest Id and WCA). This solution, called Lowest Weight, exploits their advantages and relieve to their drawbacks in terms of clusters stability and computational overhead. Simulation experiments were conducted to evaluate the performance of the algorithm proposed in terms of clusters numbers, clusterheads lifetime and the number of reaffiliations (node moving from a cluster to another). Results show that Lowest Weight ameliorate performs of existing algorithms especially regarding mobility leading to more suitable, adaptable, scalable and autonomous clustering.
\end{abstract}

\section{Introduction}

An ad hoc network is a multihop wireless network supporting cooperative mobile nodes without any existing infrastructure. In this type of networks, management tasks must be distributed over all nodes. Clustering might be an interesting technique for ad hoc networks to ensure efficiently these management tasks such as routing, addressing, transmission management and security. It consists in dividing the network into clusters managed by nodes called clusterheads. However, this technique can lead to the clusterheads congestion (processing, routing...etc). In addition, signalling messages used for executing the clustering algorithm and updating clusters can degrade the network performances. An efficient clustering algorithm must adapt itself to frequently and unpredictable topology changes known in ad hoc networks. It must also generate stable clusters as much as possible to prohibit their updates which can lead to update other information as routing, security, addressing and management information $[1,2]$.

In the literature, different works proposed clustering algorithms for ad hoc networks. These algorithms have different purposes (routing efficiency, transmission 
management, backbone formation...etc.). Our works are inspired from two existing clustering algorithms called Lowest Id [3, 4] and WCA: Weighted Clustering Algorithm [5], to propose an algorithm (Lowest Weight) that combines their strength and relieve to their limits.

WCA has the advantage of electing clusterheads based on a weight related to energy consumption, mobility, distance to neighbours and connectivity degree. However, it has drawbacks in the strategy used to divide the network into clusters since it uses a great number of clustering messages (broadcasting, many times, clustering messages in the whole network). Lowest Id minimizes this number because each node broadcasts clustering messages once and only to its one-hop neighbours. But, it uses a non suitable metric (the node identification).

The rest of the paper is organized as follows: in section 2, we present existing clustering algorithms underlying their advantages and limits. In section 3 , we describe the proposed clustering algorithm. Section 4 discusses the robustness and efficiency of our solution in comparison with existing ones. Section 5 presents simulations conducted to evaluate the performances of our algorithm. The conclusion outlines our immediate future work.

\section{State of the Art}

Many works have recently proposed clustering algorithms for ad hoc networks [3-9]. These works present advantages but some drawbacks as a high computational overhead for both clustering algorithm execution and update operations. We can classify these algorithms into two main categories: proactive algorithms and reactive ones. Most of them are proactive. Only WCA [5] is reactive. In this section, we present, in a first stage, the proactive group highlighting their advantages and drawbacks. In a second stage, we describe the only reactive algorithm (WCA). We focus our interest on clustering algorithms dealing with management tasks.

\subsection{Proactive Clustering Algorithms}

[3] and [4] describe two clustering algorithms aiming to minimize routing information and ensure efficient medium access control. In the first algorithm, called highest connectivity (CON), a node is elected as a clusterhead if it has the highest total number of one hop uncovered neighbors. Any tie is broken by the unique node identification. In the second algorithm, called lowest identification (Lowest-ID), generated clusterheads have the lowest identifications compared to their neighbors. In these two algorithms, the election of clusterheads doesn't take into consideration the quantity of energy existing in the node. Clusterheads are supposed to take in charge many energy consuming functions (routing, security, transmission management...etc) in their clusters. Unlike CON, we can note that Lowest-ID can be improved if identification will be related to energy and mobility.

A variant of CON called K-connectivity identification is described in [6] and [7] where the connectivity degree is computed on $\mathrm{k}$ hop neighbors. It generates clusters with $\mathrm{k}$ hop members. 
In [8], Nocetti, Gonzalez and Stojmenovic describe a K hop clustering algorithm called Max-Min aiming to maximize routes for fault tolerant applications. This algorithm elects clusterheads after two flooding steps called Floodmin and Floodmax. In the first flooding step, every node broadcasts k times (TTL=1) the highest node identification received. In the second step, every node broadcasts $\mathrm{k}$ times the lowest node identification received. Then, a node is elected clusterhead if it receives its ID during Floodmin otherwise it elects the minimum node ID received during the two phases as its clusterhead or it elects the maximum node ID received during the first phase as its clusterhead. Experiments show that compared to previously cited algorithm, Max-Min tends to reelect clusterheads after mobility. In addition it generates large clusters with long lifetime clusterheads. This might be an inconvenient since it drops clusterheads battery power because each one will serve a large number of nodes. In addition the election of clusterheads doesn't take into consideration the quantity of energy existing in a node. Moreover, this algorithm generates a very important overhead since it is based on $2 * \mathrm{~K}$ flooding steps.

In [9], Basagni describes a one hop clustering algorithm called DMAC (Distributed Mobility Adaptative Clustering). Nodes are elected as clusterheads based on a weight calculated on mobility and other parameters which were not specified. This algorithm is better than Lowest-ID and CON because it updates rarely its clusters structure. This might be of a great importance since updating clusterhead frequently results not only in a communication overhead to establish new clusters but also in management information updates.

\subsection{Reactive Clustering Algorithms}

Here we describe only one reactive clustering algorithm called WCA [5] (Weighted clustering algorithm). In this algorithm, each node broadcasts its weight to all nodes in the network. A node is elected as a clusterhead if it has the Lowest Weight among all uncovered nodes of the network. This process is repeated until all nodes know their roles (a clusterhead or a member). The weight used is a linear function of the node mobility, its connectivity, its consumed energy and the cumulative distance to its neighbors. An elected clusterhead serves a maximum of $\delta$ nodes. This helps it saving battery power. Moreover, the algorithm aims to build up efficient transmission management by using low power for intracluster communications and high power for intercluster communications. This minimizes energy consumption and interferences. For the update policy, the clusterhead chooses new clusterheads for its member nodes going far from it. When a node can no longer be a neighbor of any existing clusterhead, it invokes the algorithm to form new clusters. This might be very severe, especially for high mobility and it generates an important computational overhead. Moreover, information stored in the clusterheads (security, administration, policies...etc) should be reestablished after the update phase.

\section{The Proposed Clustering Algorithm}

We propose a new reactive clustering algorithm, called Lowest Weight (LW) that tries to establish efficient and stable clusters by settling down a convenient metric like 
the one specified in WCA [5] while the algorithm layout resembles Lowest-ID [3,4]. The first algorithm specifies a good metric but it generates a high computational overhead. The second algorithm minimizes the number of clustering messages exchanged to settle down clusterheads but uses a bad metric. The proposed algorithm combines the strengths of these two algorithms and specifies a new local update algorithm which minimizes the communication overhead and the nodes reconfigurations after mobility (routing tables and management information such as addressing and security). In what follows, we describe the Lowest Weight clustering algorithm. We give its basis, metric components and its design.

\subsection{Basis of Our Clustering Algorithm}

The problem of clustering can be seen as follows: given a set of nodes, how can we divide it into an optimal number of clusters without degrading the whole network performances [10]. LW aims to minimize the number of messages exchanged for both clustering and update policy to obtain a lower computational overhead. In case of mobility, it conserves a certain stability of the clusters structure. This is important to avoid re-invoking the algorithm on the whole network and losing the management information stored in the clusterheads.

\subsection{The Metric Components}

Before computing a metric, we should ask ourselves "clustering for what purpose" and "how can we minimize the generated overhead". In our solution, we aim to better manage the network. This includes all kinds of management (security, administration, transmission management, routing...etc). We can suppose that clusterheads will collaboratively ensure management tasks. To decide how much a node suited for being a clusterhead, we take into consideration the following features, inspired from the WCA metric components:

The Battery Power (BP): Compared to ordinary nodes, clusterheads ensure some services. Thus, we should elect nodes with highest remaining battery power as clusterheads. In WCA, this metric component is computed as the cumulative time Pv, during which a node $\mathrm{v}$ acts as a clusterhead. Pv implies how much battery power has been consumed. We have opted to consider the remaining battery power because, in ad hoc networks, at the network bootstrapping, nodes can have different quantities of energy.

The mobility (M): We aim to have stable clusterheads. So we should elect nodes with low mobility as clusterheads. Unlike WCA which computes M from the network bootstrapping till current time $\mathrm{T}$, we compute it as the average speed for the last period of time $\mathrm{P}$, from $\mathrm{T}-\mathrm{P}$ till current time $\mathrm{T}$ as show in equation (1):

$$
M=\frac{1}{p} \sum_{t=T-P}^{T} \sqrt{\left(X_{t}-X_{t^{\prime}}\right)^{2}+\left(Y_{t}-Y_{t^{\prime}}\right)^{2}}
$$

Where $\left(X_{t}-X_{t}\right)$ and $\left(Y_{t}-Y_{i}\right)$ are the coordinates of the node at time $\mathrm{t}$ and t'. 
We preferred this way to compute $\mathrm{M}$ because a node can switch from high (respectively lower) mobility to very lower (respectively very high) one. The node coordinates can be estimated as described in [11].

The Node Connectivity Degree (C): we shouldn't elect nodes with highest connectivity or lowest one as clusterheads. In fact, in the first case, they will be congested and their battery power will drop rapidly. In the second case, the clusters size will be very low and we won't take advantage of clustering. Hello messages are used to compute $\mathrm{C}$. In fact, each node broadcasts a hello message with TTL=1 (including its identification) then uses received hello messages to compute its connectivity degree $\mathrm{C}$.

The Distance to Neighbours (D): it's better to elect a clusterhead with the nearest members. This might minimize node detachments. For a node v, D is computed as the cumulative mean square distance to neighbors divided by the total number of neighbors as shown in equation (2):

$$
D=\frac{1}{C} \sum_{v^{\prime} \in N(v)} \sqrt{\left(X_{v}-X_{v^{\prime}}\right)^{2}+\left(Y_{v}-Y_{v^{\prime}}\right)^{2}}
$$

where $\left(X_{v} Y_{v}\right)$ and $\left(X_{v^{\prime}} Y_{v^{\prime}}\right)$ are the coordinates of the node $\mathrm{v}$ and $\mathrm{v}$ respectively and $\mathrm{N}(\mathrm{v})$ is v's list of neighbors. These coordinates can be estimated as described in [11]. We assume that this method for computing D is more efficient than the one used in WCA where D is just the cumulative distance to neighbors. In fact, a node with a high number of neighbors close to it can have a distance superior than the one of a node with very few neighbors which are far from it.

\subsection{The Proposed Algorithm}

LW combines each of the above parameters with certain weighing factors chosen according to the application needs and various networks environment (battlefield, conferencing, vehicular applications...etc). The algorithm is executed for only one time (at the system bootstrapping). Then the updating procedure is locally invoked after mobility or to attach new nodes joining the network. First, we describe clusterheads election procedure. Then, we present the update policy.

\section{Clusterhead Election:}

Messages and notations used in the algorithm are described in table 1. Each node:

- Broadcasts a hello message with TTL=1 (including its identification).

- Uses received hello messages to compute its connectivity degree $C$ (total number of neighbors) then computes its weight as shown in equation (3).

$$
\begin{aligned}
& W=w_{1} \times B P+w_{2} \times M+w_{3} \times C+w_{4} \times D \\
& \text { where } w_{1}+w_{2}+w_{3}+w_{4}=1
\end{aligned}
$$

- Broadcasts its weight to its one hop neighbors (TTL=1)

- Computes a list called neighbor list (NL) that contains the identifications of neighbor nodes and their corresponding weights NL. 
If the node possesses the minimum weight compared to the weights in NL it proclaimed itself clusterhead by sending a role message UPDATE_ROLE (My_ID, My_ID) to its one hop neighbors.

If it isn't the case, it expects role messages from its neighbors with lower weights than its one. If it receives at least one role message as clusterhead, it chooses the Lowest Weight node among them. It broadcasts a role message UPDATE_ROLE (My_ID, My_CH_ID) to confirm its role as an ordinary node and attach itself to that clusterhead, identified by My_CH_ID. Otherwise, it becomes a clusterhead and broadcasts a role message UPDATE_ROLE (My_ID, My_ID) to its one hop neighbors.

Table 1. Messages exchanged and notations

\begin{tabular}{|c|c|}
\hline Message/notation & Meaning \\
\hline My_ID & The identification of the node that sends the message \\
\hline My_CH_ID & $\begin{array}{l}\text { The clusterhead identification of the node that sends the message } \\
\text { (My ClusterHead IDentification). }\end{array}$ \\
\hline $\begin{array}{l}\text { UPDATE_ROLE } \\
\text { (My_ID, My_CH_ID) }\end{array}$ & $\begin{array}{l}\text { A node sends an update message to declare its role as an ordinary } \\
\text { node and attach itself to the clusterhead identified by } \mathrm{My} \_\mathrm{CH} \text { IID. }\end{array}$ \\
\hline $\begin{array}{l}\text { UPDATE_ROLE } \\
(\text { My_ID, My_ID) }\end{array}$ & A node sends an update message to declare its role as a clusterhead. \\
\hline
\end{tabular}

\section{Update Policy}

The update policy process begins after the election procedure. In our algorithm, we suppose that clusterheads broadcast periodically hello messages. In what follows, we describe how our approach reacts to topology changes.

When an ordinary node moves: When an ordinary node moves within its corresponding cluster (that means that it can join its clusterhead), no changes occurs. Otherwise, the moving node can leave its cluster. Thus, it can find itself closer to multiple or none clusterheads (declared in its neighboring list). In the first case, it chooses the Lowest Weight clusterhead as its new clusterhead and broadcasts its new role as UPDATE_ROLE (My_ID, My_CH_ID). For the second case, the node executes locally the clustering algorithm by sending a hello message to its uncovered neighbours.

When a clusterhead moves: When a clusterhead moves, it leaves its role as a clusterhead until it detects one or numerous clusterheads in its neighborhood. If it has the Lowest Weight, it keeps its role as it is; otherwise it becomes an ordinary node and attaches itself to the Lowest Weight clusterhead by broadcasting an update message UPDATE_ROLE (My_ID, My_CH_ID).

\section{An Illustrative Example}

We explain our clustering algorithm execution by applying it on the set of nodes described in Fig 1. Nodes are represented by circles dotted with their identifications. An edge between two nodes exists if each one is on the sight of the other. All existing edges are associated with the corresponding distance between the corresponding two nodes. Table 2 shows, for each node, its metric components as well as the corresponding weight. The two metrics BP and M are arbitrary chosen. The weight factors considered are $\mathrm{w} 1=0.2, \mathrm{w} 2=0.3, \mathrm{w} 3=0.2$ and $\mathrm{w} 4=0.3$. 
Table 2. Weight computations for each node

\begin{tabular}{cccccc}
\hline Node ID & C & D & BP & M & Weight \\
\hline 1 & 4 & 2.5 & 2.1 & 2.33 & 2.436 \\
2 & 5 & 3.4 & 2.9 & 1.25 & 2.85 \\
3 & 2 & 2.5 & 3.6 & 5.14 & 2.898 \\
4 & 2 & 4 & 5.5 & 2.23 & 3.146 \\
5 & 2 & 1 & 6.3 & 4.8 & 2.92 \\
6 & 4 & 2.5 & 2.1 & 1.78 & 2.32 \\
7 & 2 & 2.5 & 3.14 & 5.58 & 2.894 \\
8 & 4 & 1.75 & 5.21 & 3.28 & 3.023 \\
9 & 2 & 4 & 7.12 & 5.23 & 4.07 \\
10 & 3 & 2 & 8.4 & 2.75 & 3.43 \\
11 & 4 & 2.5 & 2.7 & 2.14 & 2.518 \\
\hline
\end{tabular}

Fig 2 shows the clusters identified. In fact, the nodes 1, 6 and 11 declare themselves as clusterheads because each one has the minimum weight compared to the weights of its one hop neighbors. Node 2 chooses 6 as its clusterhead from the set $\{6$, 1 and 11 \} because 6 have the Lowest Weight...etc.

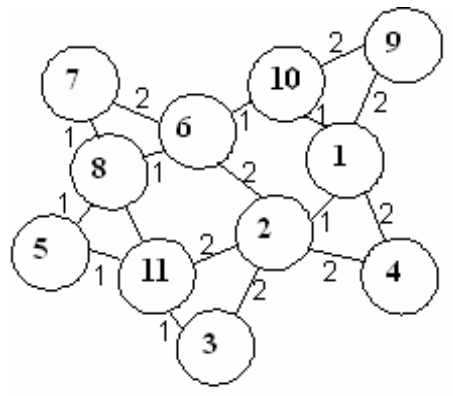

Fig. 1. Nodes with corresponding neighbors and distances

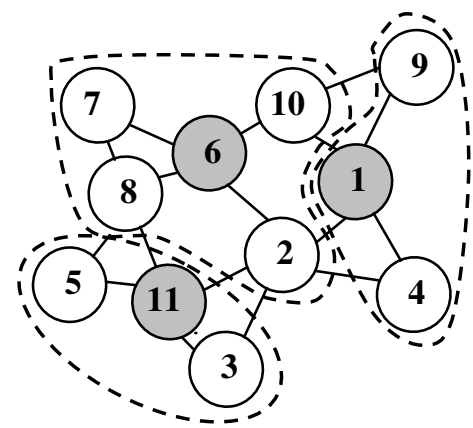

Fig. 2. Clusters identified

\section{Main Contributions}

Unlike the clustering algorithms described above [3, 4, 6-8], our solution uses a suitable metric since it takes into account the quantity of energy existing in the node and its mobility. Thus, it generates more stable and durable clusterheads. In addition, unlike those solutions, our approach is reactive and it specifies a local update phase while those solutions are proactive. A proactive algorithm is re-invoked periodically even if there's no change in the network topology. This can result in useless exchanged clustering messages and can then degrade the performances of the system. 
In comparison with WCA, our approach generates a lower overhead. In fact, each node has to broadcast (TTL=1) only 3 messages to know its role( a hello message for neighbors discovery, a weight message and a role message to declare its role). In WCA [5], each node has to broadcast in the whole network o $\left(n^{2}\right)$ messages to know its role ( $\mathrm{n}$ is the number of nodes in the network). Moreover, during the algorithm computation, the non covered nodes must re-compute their weights and re-diffuse them. This can lead to a high computational overhead. Furthermore, to decide its role, a node must compare its weight to all weights in the network. But, how does a node know that it received all weights? Thus, WCA might not converge.

We can say also that our approach uses a more adequate update policy. In fact, unlike WCA, we update locally the clusters structure. Moreover, the clustering algorithm is just applied during the network bootstrapping. Then the update phase is executed by each node if it detects special changes in its neighborhood. In WCA, the update phase consists in reapplying the clustering algorithm when a node, can't be attached to any clusterhead. This might change all clusters structure. In fact, all management policies (configurations, security, etc) could be lost and nodes will be obliged to re-establish them.

\section{Simulation Experiments}

To study our proposed solution and compare its performances to other clustering algorithms, we have extended the NS2 simulator so that it permits to support clustering techniques. Five algorithms were implemented: Lowest-ID, CON, CON-ID, WCA and LW. We focused our study on our proposed algorithm and compare its results essentially to WCA that presents better performances than Lowest-ID.

We fixed three main performance criteria which are:

- clusterheads lifetime

- node reaffiliations

- average number of clusters

These parameters are studied by varying nodes number transmission range and maximum node speed.

The scenarios were generated using the random waypoint model with input parameters such as maximum speed, pause times, number of nodes, area and simulation period. The simulation parameters are listed in Table 3 . The weight values used for simulation are $\mathrm{w} 1=0.7, \mathrm{w} 2=0.2, \mathrm{w} 3=0.05$ and $\mathrm{w} 4=0.05$.

Table 3. Simulation Parameters

\begin{tabular}{lll}
\hline \multicolumn{1}{c}{ Parameter } & \multicolumn{1}{c}{ Meaning } & \multicolumn{1}{c}{ Value } \\
\hline $\mathrm{N}$ & Number of nodes & $20-60$ \\
Grid $(\mathrm{m} \times \mathrm{n})$ & Scenario area & $100 \times 100 \mathrm{~m}^{2}$ \\
$\mathrm{Tx}$ & Transmission range & $10-70 \mathrm{~m}$ \\
& & $10-120 \mathrm{~m}$ \\
PauseT & Pause time & $0 \mathrm{sec}$ \\
MaxSpeed & Maximum speed of nodes & $1-10 \mathrm{~m} / \mathrm{s}$ \\
\hline
\end{tabular}




\subsection{Discussion of Results}

We will discuss results while varying transmission range in a first step and mobility in a second step.

\section{Results for Varying Transmission Range (Tx)}

Fig 3 shows, for varying nodes number, the variation of the average number of clusterheads with respect to the transmission range for both LW (a) and WCA (b). The maximum speed was fixed to $5 \mathrm{~m} / \mathrm{s}$. We notice that the average number of clusterheads decreases with the increase in the transmission range. In fact, a clusterhead with a large transmission range will cover a larger number of nodes.

[12] has shown that an optimum decomposition of a network of $\mathrm{n}$ nodes into clusters should be $\sqrt{n}$ clusters of $\sqrt{n}$ members each one. We notice that both LW and WCA give a good clustering of the network. However, for the same simulation parameters, our results are close to that optimum $(\sqrt{n})$. For example, for $\mathrm{n}=60$ and $\mathrm{Tx}=40$, WCA generates 6 clusters while LW generates 7.5 clusters (the optimum for $\mathrm{n}=60$ is 7.74 )

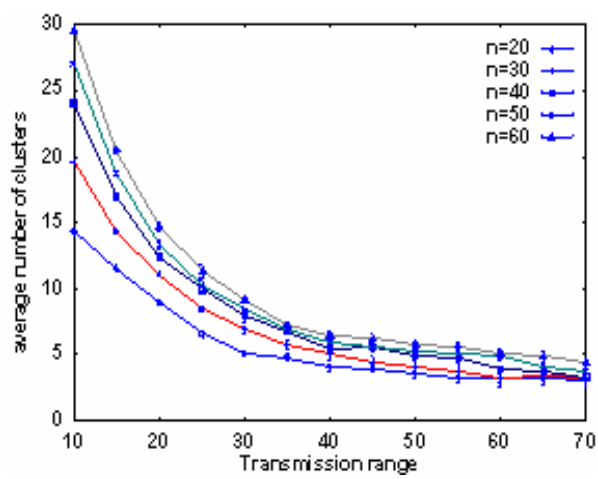

(a) LW

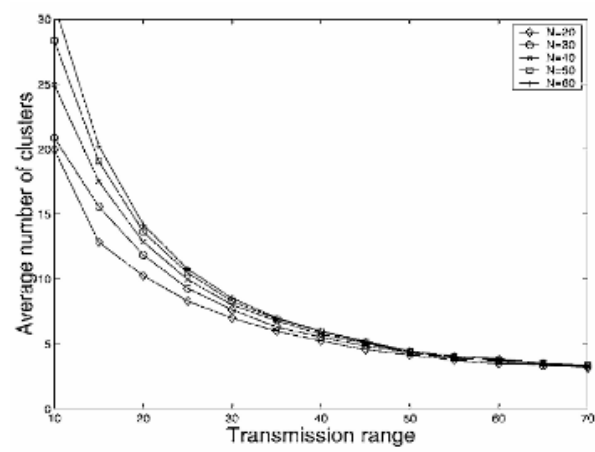

(b) WCA

Fig. 3. LW and WCA average number of clusterheads, max_speed $=5$

Fig 4 shows the reaffiliations per unit time with respect to the transmission range, where maximum speed is $5 \mathrm{~m} / \mathrm{s}$. We notice that reaffiliations increases with the increase of transmission range, reaches a peak where transmission range is around $65 \mathrm{~m}$, then decreases. This behavior could be explained as follows: for lower transmission range, there are many clusters and the nodes are closer to their clusterheads. Then, while the transmission range increases, clusterheads cover much more moving nodes which can leave the corresponding clusters. After the peak, a clusterhead still cover a large number of nodes which, in spite of their movement, stay in the large area covered by the clusterhead.

In comparison with WCA results, we observe that Lowest Weight results in more stable clusters as it yields as much as $75 \%$ reduction in the rate of reaffiliations per unit time. This reduction shows that LW uses a more efficient metric and update procedure than WCA. 


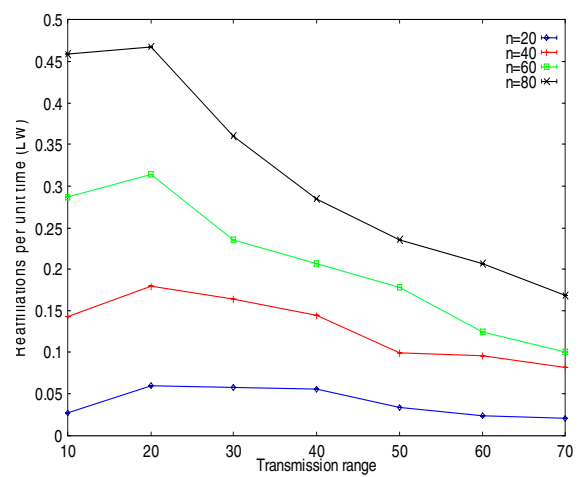

(a) $\mathrm{LW}$

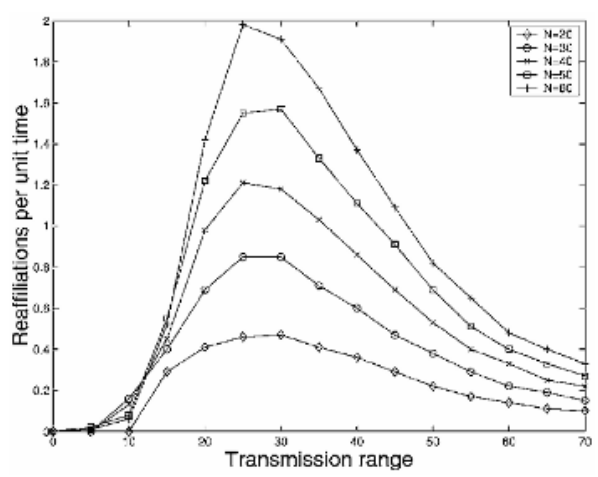

(b) WCA

Fig. 4. LW and WCA average number of reaffiliations per unit time, max_speed = 5

Fig 5 shows, clusterheads lifetime with respect to the transmission range where maximum speed is $5 \mathrm{~m} / \mathrm{s}$. We observe that clusterheads lifetime increases with the increase in the transmission range. This is because, for nodes with higher transmission range, the number of clusterhead decreases and the elected clusterheads are far from each other. Thus, the probability that a moving clusterhead becomes a neighbor of another one is minimized.

\section{Results for Varying Mobility}

Fig 5 and 6 show the variation of the same metrics but for varying the nodes maximum speed from $1 \mathrm{~m} / \mathrm{s}$ to $10 \mathrm{~m} / \mathrm{s}$. In these scenarios, the transmission range is fixed to $30 \mathrm{~m}$ like in WCA simulations.

Fig 5 (a) shows that, for LW, the average number of clusterheads is almost the same with respect to the maximum speed of nodes. In WCA (Fig 5 (b)), we observe that the number of clusterheads decreases slightly with respect to maximum speed.

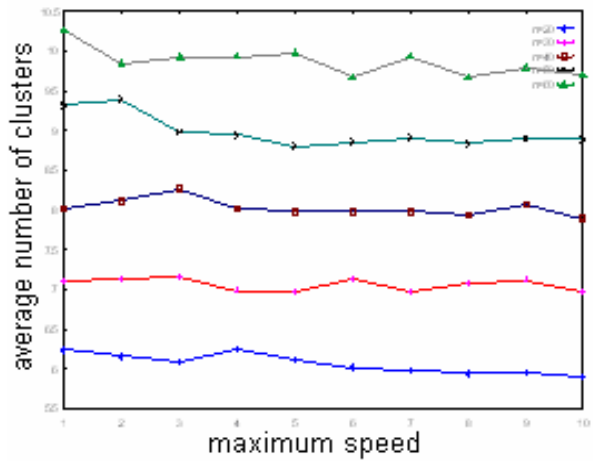

(a) $\mathrm{LW}$

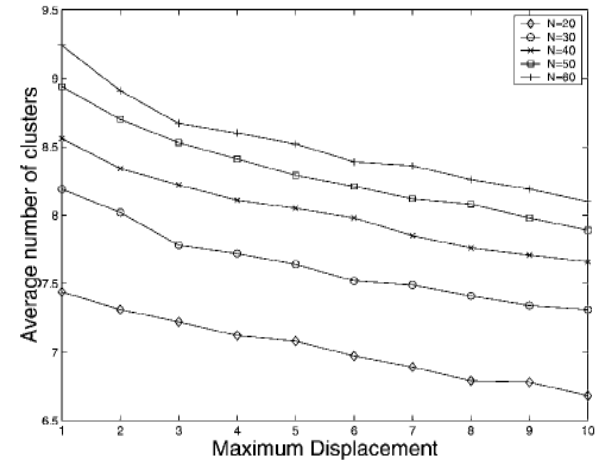

(b) WCA

Fig. 5. LW and WCA average number of clusters, $T x=30 \mathrm{~m}$ 
Fig 6 shows the reaffiliations per unit time with respect to the maximum nodes speed where. We notice that reaffiliations increase with the increase of nodes speed. This is because nodes with higher speed quit rapidly their cluster to reach another one.

In comparison with WCA, we observe that our algorithm results in more stable clusters as it yields as much as $60 \%$ reduction in the rate of reaffiliations per unit time. In addition, [5] shows that WCA presents the minimum reaffiliation rate compared to Lowest-ID [3, 4], CON [3, 4], CON-ID [6, 8], DCA and DMAC [9]. Compared to WCA results (Fig 6 (b)), we observe that LW generates more stable clusters.

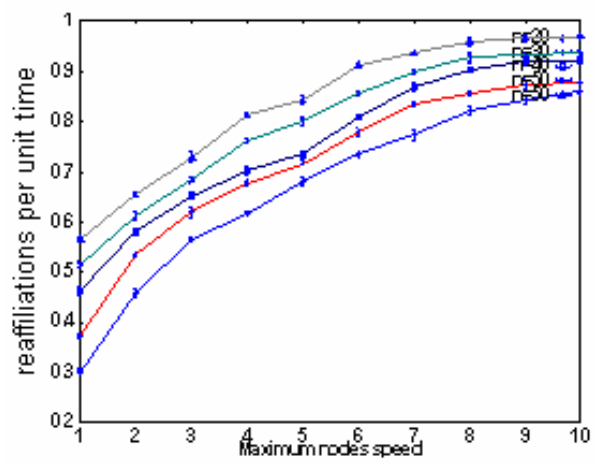

(a) $\mathrm{LW}$

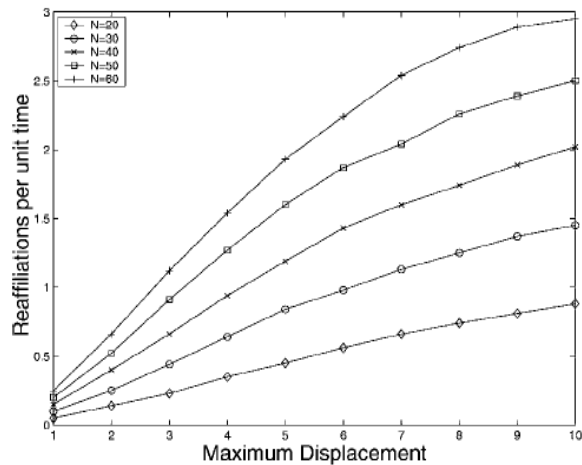

(b) WCA

Fig. 6. Reaffiliations per unit time, $T x=30 \mathrm{~m}$

\section{Conclusions}

In this paper, we have proposed a reactive clustering algorithm for ad-hoc networks called Lowest Weight. This algorithm is inspired from two existing algorithms lowest-ID and WCA avoiding their limits. In LW, we have exploited the good strategy of Lowest-ID for clustering. This strategy takes into account only neighbors of a given node while WCA considers the entire network which leads to a great number of clustering messages exchanged between nodes. In selecting clusterheads, we have opted for a metric inspired from the WCA one (the node's degree, mobility, remaining energy and cumulative distance to neighbors). However, we have defined an update procedure that is proper to $\mathrm{LW}$.

We have conducted simulations to evaluate the performances of the LW and compared results essentially to WCA that presents better performances than Lowest-ID. Experiments have shown that LW gives a better clustering than WCA as the number of generated clusterheads is close to the optimum $\sqrt{n}$. In addition, LW results in more stable clusters. Indeed, it allows $30 \%$ reduction in the reaffiliation rate per unit time while varying transmission range and $60 \%$ while varying mobility.

Our future works will be focused on testing a distributed Public Key Infrastructure over an ad-hoc network structured by the Lowest Weight algorithm. 


\section{References}

1. S.Sivavkeesar, G.Pavlou, A.Liotta, «Effective management through Prediction base clusering approach in the next generation adhoc networks »

2. M.E.Elhdhili, L.BenAzzouz, F.Kamoun, 2004 " A Totally Distributed Cluster Based Key Management Model for Ad hoc Networks”. Proc. Med-Hoc-Net 3, pp. 291-299.

3. M. Gerla and J.T.-C. Tsai, 1995,"Multicluster, mobile, multimedia radio network", ACM/Baltzer Journal of Wireless Networks. vol. 1, (no. 3), pp.255-265.

4. C.R. Lin and M. Gerla. 1997, "Adaptive clustering for mobile wireless networks". IEEE Journal on Selected Areas in Communications, Vol. 15, No. 7, pp. 1265-1275.

5. M. Chatterjee, S. K. Das, and D. Turgut, 2002, "WCA : A weighted clustering algorithm for mobile ad hoc networks", ClusterComputing 5, pp. 193-204.

6. G. Chen, F. G. Nocetti, J. S. Gonzalez, and I. Stojmenovic. 2002, "Connectivity Based khop Clustering in Wireless Networks". In 35th Hawaii International Conference on System Sciences.

7. A. D. Amis, R. Prakash, T. H.P. Vuong and D. T. Huynh, 2000, "Maxmin Dcluster formation in wireless ad hoc networks," Proc. IEEE Infocom pp. 32-41.

8. F. G. Nocetti, J. S. Gonzalez, and I. Stojmenovic, 2003, "Connectivity Based k-hop Clustering in Wireless Networks." Telecommunication Systems 22, pp. 205-220.

9. S. Basagni, 1999, "Distributed and mobility-adaptive clustering for multimedia support in multi-hop wireless networks". Proceedings of the IEEE Vehicular Technology Conference (VTC), Amsterdam, The Netherlands, pp. 19-22.

10. Charles E. Perkins, ad hoc networking, Addison-Wesley Pub Co, 1st edition December 29, 2000.

11. C. Bettstetter, Giovanni Resta and Paolo Santi, 1993,"The Node Distribution of the Random Waypoint Mobility Model for Wireless Ad Hoc Networks," IEEE Trans. Mobile Computing, vol.2, no.3, pp.257-269.

12. L. Kleinrock and F. Kamoun, Hierarchical routing for large networks Performance evaluation and optimization, Computer networks, pp155-174, 11177 